\title{
Pulmonary AngII promotes LPS-induced lung inflammation by regulating microRNA-143
}

\author{
Shenglan Wang $\cdot$ Yan Tan $\cdot$ Tingting Yang $\cdot$ Chen Liu $\cdot$ Rufang Li
}

Received: 28 October 2020/Accepted: 27 August 2021 / Published online: 3 September 2021

(C) The Author(s), under exclusive licence to Springer Nature B.V. 2021

\begin{abstract}
Angiotensin converting enzyme 2 (ACE2) is a terminal carboxypeptidase, which cleaves single terminal residues from several bioactive peptides such as Angiotensin II (AngII). Many investigations indicated that ACE2 functions in angiotensin system and plays a crucial role in inflammatory lung diseases. However, the mechanism behind the involvement of ACE2 in inflammatory lung disease has not been fully elucidated. In this study, BEAS-2B cells were treated with gradient concentration of AngII and lipopolysaccharide (LPS) to induce inflammatory condition. Quantitative RT-PCR was performed to detect the level of ACE2 and miR-143-3p. Western blotting and immunofluorescence assays were performed to measure the expression of related proteins. The levels of inflammatory cytokines and cell viability were respectively measured by ELISA and CCK- 8 kits. And ACE2 activity was detected by corresponding
\end{abstract}

Shenglan Wang and Yan Tan make equal contributions for this work.

S. Wang $(\bowtie) \cdot$ Y. Tan $\cdot$ T. Yang $\cdot$ C. Liu $\cdot$ R. Li

Pulmonary and Critical Care Medicine, The First People's

Hospital of Yunnan Province, 157 Jinbi Road, Xishan

District, Kunming 650032, Yunnan, China

e-mail: Wangshenglanzz@163.com

S. Wang $\cdot$ Y. Tan $\cdot$ T. Yang $\cdot$ C. Liu $\cdot$ R. Li

The Affiliated Hospital of Kunming University of Science and Technology, 157 Jinbi Road, Xishan District,

Kunming 650032, Yunnan, China commercial kits. Bioinformatics methods were employed to predict the potential microRNA targeting ACE2, which was then confirmed by dual luciferase reporter assay. The results showed that ACE2 expression and activity were time-dependently decreased in LPS group for the first $12 \mathrm{~h}$, after which this tendency was reversed. AngII addition enhanced these effects, compared with LPS group. Additionally, the lowest ACE2 activity level was found in both LPS and AngII + LPS groups at $6 \mathrm{~h}$. The number of nuclei and the ACE2 expression were decreased in LPS groups at $6 \mathrm{~h}$ and further reduced by addition of AngII, detected by immunofluorescence. Moreover, ACE2 was validated to be a direct target of miR-143-3p. Pretreatment of AngII and LPS regulated the activity of ACE2, increased the expression of proinflammatory cytokines and cell apoptosis and regulated the expression of Bax, Bcl-2 and cleaved caspase-3 in BEAS-2B cells, which could be reversed by transfecting miR-143-3p inhibitor. The results collectively suggest that AngII promotes LPS-induced inflammation by regulating miR-143-3p in BEAS-2B cells. Therefore, miR-143$3 p$ is considered a potential molecular target for the treatment of lung inflammation.

Keywords ACE2 - AngII - MicroRNA-143 · Inflammation $\cdot$ Bronchial epithelial cell 


\section{Introduction}

Lung inflammation is a nonnegligible pulmonary response to many kinds of external stimulation (Khair et al. 1996). It, as is known, can be caused by pneumonias, sepsis, immune deficiency or trauma. However, the common instigating events remain unknown (Li et al. 2011). Lung inflammation usually occurs with pathogen exposure, which is often associated with molecular patterns such as lipopolysaccharide, LPS (Boskabadi et al. 2019). Acute LPS exposure is a trigger of respiratory symptoms or inflammation, which can be observed in vitro as increased levels of pro-inflammatory cytokines and neutrophil infiltration (Arora et al. 2011; Hu et al. 2019). The investigation of lung inflammation mechanism should be proceeded and deepened, which is a must for discovering unique and effective therapeutic targets for lung inflammation.

The role of Angiotensin II (AngII) has been reported in the development of atherosclerosis and in the arterial response of injury and restenosis. And the mechanisms above all include extracellular matrix production, cytokine induction and vascular hypertrophy (Silva et al. 2020). The angiotensin-converting enzyme (ACE) is a metallo monocarboxypeptidase, the only one of several enzymes that can produce AII and is generally considered as a component of the renin angiotensin system (Zhang et al. 2015). The participation of ACE2 in lung function has attracted research attention in recent years because of the findings supporting that this ectoenzyme is also the receptor of the SARS and NL63 human coronaviruses, CoVs ( $\mathrm{Li}$ et al. 2003). Moreover, the discord of pulmonary ACE2 function plays an important role in respiratory diseases including severe infections such as SARS-CoV, sepsis and acid aspiration (Sodhi 2018). Recently, some studies found that miRNAs were closely associated with the pathogenesis of coronavirus disease (Matarese et al. 2020; Tang et al. 2020; Gallicano et al. 2020).

However, the role and the potential mechanism of pulmonary ACE2 in inflammation are still unknown and in need of exploration. The recognition of differently expressed miRNAs through bioinformatics database and transcriptome data could help us to understand and investigate pathological mechanisms in sepsis and associated lung injury (Ahmad et al. 2019, 2020). A study report that miRNA-34a inhibition was able to improve lung injury and reduce the levels of inflammatory markers in LPS-induced lung injury (Khan et al. 2020). MicroRNAs (miRs) are approximately 22 nucleotides RNA molecules which regulate gene expression at the posttranscriptional level by escalating mRNA degradation or translational repression (Kim et al. 2009). miRs participate in most basic biological processes, whose abnormal expression has been found in many human diseases (Bartel and Bartel 2009).

miR-143/145 was previously reported to be expressed in cardiomyocytes and smooth muscle cells, SMCs (Wang et al. 2014). A study demonstrated that inhibiting ACE and/or angiotensin type 1 (AT1) receptor in mice with loss of miR-143/145 rescued the vascular dysfunction and the aberrant gene expression (Boettger et al. 2009). Further, it is known that miR-143 has close contact with human immune response and may become in future a therapeutic target for immunization in view of its adjustment for the anti-inflammatory effect of mesenchymal stem cells (Ponsuksili et al. 2017). According to the latest study, miR-143 is intimately related to lung sepsis in mouse, and human leukocytes after inducement of LPS (Han et al. 2016). However, little is known about the relationship between ACE2 and miR-143 in the lung immune response. The purpose of the present study was to investigate the exact role of AngII in lung inflammation, and further elucidate the underlying mechanism.

\section{Materials and methods}

Cell culture and reagent

The human bronchial epithelial cells BEAS-2B were acquired from the American Type Culture Collection (Manassas, VA, USA). Cells were cultured in Dulbecco's modified Eagle's medium/Ham's Nutrient Mixture F12 (DMEM/F12, Gibco, Life Technologies, USA) supplemented with $10 \%$ fetal bovine serum (FBS, Gibco, Life Technologies) and 1\% penicillinstreptomycin-glutamine (Gibco, Life Technologies). Flask was placed at $37{ }^{\circ} \mathrm{C}$ in a humidified incubator (Thermo Fisher Scientific, USA) with $5 \% \mathrm{CO}_{2}$.

LPS and AngII were purchased from SigmaAldrich (St Louis, MO, USA). The serum-free DMEM/F12 was used to dilute LPS to $1 \mathrm{mg} / \mathrm{L}$, and 
AngII solution to $1,5,10$ or $50 \mu \mathrm{mol} / \mathrm{L}$ before experiments. BEAS-2B cells were treated with $1 \mathrm{mg} /$ L LPS for $24 \mathrm{~h}$, and/or $0,1,5,10$ or $50 \mu \mathrm{mol} / \mathrm{L}$ AngII every $24 \mathrm{~h}$ for $96 \mathrm{~h}$ in this research.

\section{Cell transfection}

One day prior to transfection, BEAS-2B cells $\left(5 \times 10^{4}\right.$ cells/well $)$ were seeded into 6-well plates and cultured at $37{ }^{\circ} \mathrm{C}$ in an atmosphere containing $5 \%$ $\mathrm{CO}_{2}$. Subsequently, the cells were transfected with small interference RNA targeting miR-143-3p mimic, miR-143-3p inhibitor or negative control (NC) using Lipofectamine 3000 (Invitrogen; Thermo Fisher Scientific, Inc.), according to the manufacturer's protocol. Cells of the blank control group (control) did not receive any treatment. Following incubation for $48 \mathrm{~h}$, these cells were collected for the following experiments. Transfection efficiency was detected by reverse transcription-quantitative polymerase chain reaction (RT-qPCR).

\section{Real-time quantitative PCR}

qRT-PCR was performed to detect the expression level of miR-143-3p and ACE2 in BEAS-2B cells after corresponding treatment or transfection. Briefly, total RNAs in BEAS-2B cells were extracted using TRIzol Plus RNA Purification kit (Invitrogen). cDNA was reversely transcribed using SuperScript Reverse Transcription Reagents (Invitrogen). The expression level of miR-143-3p and ACE2 were then measured at 0, 3, 6, 12 and $24 \mathrm{~h}$ after AngII and/or LPS treatment using TaqMan MicroRNA Assay (Applied Biosystems), with U6 as the endogenous control. The primer sequences were as follows: miR-143-3p forward: $5^{\prime}-$ CTGGCGTTGAGATGAAGCAC-3', and reverse: $5^{\prime}$ CAGAGCAGGGTCCGAGGTA-3'; ACE2 forward: 5'-TCTGCCACCCCACAGCTT-3', and reverse: $5^{\prime}$ GGCTGTCAAGAAGTTGTCCATTG-3'; U6, forward: 5'-GCGAGCACAGAATTAATACGAC- ${ }^{\prime}$, and reverse: $5^{\prime}$-AACGCTTCACGAATTTGCGT- ${ }^{\prime}$. Data were quantified using the classic $2^{-\Delta \Delta \mathrm{Ct}}$ method (Livak and Schmittgen 2001).

Cell viability assay

Viability of BEAS-2B cells was evaluated using cell counting kit-8 (CCK-8) assay (Beyotime
Biotechnology, Shanghai, China) in this research. Briefly, BEAS-2B cells were seeded into 96-well plates $\left(2 \times 10^{3}\right.$ cell/well) respectively after AngII ( 1 , $5,10$ or $50 \mu \mathrm{mol} / \mathrm{L})$ and/or LPS ( $1 \mathrm{mg} / \mathrm{L})$ treatment. And $10 \mu \mathrm{L} \mathrm{CCK}-8$ solution was added into each well followed by incubation at $37{ }^{\circ} \mathrm{C}$. Subsequently, the absorbance of each well was recorded at $450 \mathrm{~nm}$ after $24 \mathrm{~h}, 48$ and $96 \mathrm{~h}$ of incubation using Micro-plate Reader (Bio-Tek Instruments, Winooski, VT, USA). Cell viability (\%) was calculated as the ratio of average absorbance of treatment group/average absorbance of control group $\times 100 \%$.

\section{Measurement of ACE2 activity}

Measurement of ACE2 activity (SensoLyte 390 ACE2 activity assay kit; AnaSpec, USA) was conducted in accordance with the supplied manual. Briefly, cells were incubated for $15 \mathrm{~min}$ at $4{ }^{\circ} \mathrm{C}$ and then centrifugated at $20,000 \times \mathrm{g}$ for $10 \mathrm{~min}$. The supernatants were collected and stored at $-80{ }^{\circ} \mathrm{C}$ for ACE2 activity measurement. Total protein concentration was quantified using the Bradford protein assay.

Immunofluorescence

BEAS-2B cells were incubated with polyclonal antisera against ACE-1 (catalog ab39172; Abcam). Secondary antibodies used were appropriate fluorescent labeled secondary antibodies (1:1000, Life Technologies, Inc.) as well as the nuclear marker DAPI (Biolegend). The slides were then mounted using Gelvatol (Sigma-Aldrich) solution prior to imaging using a Zeiss LSM 710 confocal microscope (Carl Zeiss, Jena, Germany) under appropriate filter sets.

\section{Enzyme-linked immunosorbent assay (ELISA)}

In supernatant samples, IL-1 $\beta$, IL-6 and tumor necrosis factor $\alpha$ (TNF- $\alpha$ ) were measured using ELISA kits (R\&D Systems, Minneapolis, MN, USA) according to the manufacturer's instructions.

Western blot analysis

The AngII and/or LPS-induced cells were collected and lysed at $24 \mathrm{~h}$ after treatment using RIPA buffer. Identical amounts of proteins were resolved by 10-15\% SDS-PAGE and then transferred to a PVDF 
membrane (Millipore Corporation, Billerica, MA, USA). The membrane was incubated with specific antibodies (Bcl-2, Bax, cleaved caspase3, ACE2 and $\beta$-actin; Cell Signaling Technology, Inc.), and peroxidase-conjugated secondary antibodies (Gaithersburg, MD, USA) at $4{ }^{\circ} \mathrm{C}$ overnight. Chemiluminescence (Millipore Corporation) and densitometry analysis (ImageJ software) were applied to the protein expression detection.

\section{Dual luciferase reporter assay}

It was predicted by bioinformatics software (http:// www.targetscan.org) that ACE2 can be a potential target of miR-143-3p. To confirm this prediction, the 3 -UTR of ACE2 containing the miR-143-3p binding site was cloned into pmirGLO dual luciferase miRNA reporter vectors (Promega, Madison, WI, USA). A mutated $3^{\prime}$-UTR of ACE2 was introduced into the potential miR-143-3p binding site. The reporter vectors containing the wild type or mutant of ACE2 3'UTR and miR-143-3p mimic or miR-NC were cotransfected into BEAS-2B cells using Lipofectamine 3000 (Invitrogen). The relative luciferase activity was detected right after $48 \mathrm{~h}$ of transfection with a dualluciferase reporter assay system (Promega Corporation, Madison, WI, USA).

Statistical analysis

Data are expressed as the mean \pm standard deviation. SPSS 17.0 statistical software (SPSS, Inc., Chicago, IL, USA) was used for all statistical analyses. Comparisons between groups were analyzed by Student's t-test or one-way analysis of variance followed by Tukey's test. $\mathrm{P}<0.05$ is considered to indicate a statistically significant difference.

\section{Results}

ACE2 is up-regulated in BEAS-2B cells treated with AngII in low concentrations

To investigate the role of ACE2 and AngII in lung inflammation, we measured ACE2 expression in the human bronchial epithelial cells. CCK-8 assay showed that AngII $(0,1,5,10$ or $50 \mu \mathrm{mol} / \mathrm{L})$ significantly reduced cell growth and viability of BEAS-2B in a dose-dependent manner after $24 \mathrm{~h}$ (Fig. 1A). As shown by RT-qPCR analysis (Fig. 1B), the expression level of ACE2 was increased most significantly by 5 $\mu \mathrm{mol} / \mathrm{L}$ AngII compared with the control group without AngII treatment and other groups treated with different concentrations of AngII. Therefore, we chose $5 \mu \mathrm{mol} / \mathrm{L}$ AngII for our further studies.

AngII promotes LPS-induced inflammation in BEAS-2B cells

Next, we used LPS to further investigate AngII biology and its possible role in mediating inflammation. Compared with the control group without LPS and AngII, $1 \mathrm{mg} / \mathrm{L}$ LPS significantly reduced cell viability of BEAS-2B, which could be further reduced by adding AngII (Fig. 2A). Moreover, we examined whether the ACE2 activity was altered by LPS and AngII in BEAS-2B cells using the SensoLyte 390 ACE2 activity assay kit. As shown in Fig. 2B, ACE2 activity was time-dependently decreased in LPS group within $12 \mathrm{~h}$ compared with control group, and this tendency could be reversed after $12 \mathrm{~h}$. By adding AngII, the effects mentioned above could be enhanced compared with LPS group. As shown by RT-qPCR analysis (Fig. 2C), after interfering with LPS, the expression level of ACE2 mRNA was significantly increased in the first $3 \mathrm{~h}$, then decreased from 3 to $12 \mathrm{~h}$, and noticeably increased again after $12 \mathrm{~h}$. After pretreatment with AngII, the increasing ACE2 was significantly receded in $3 \mathrm{~h}$ compared with LPS group. And from 3 to $12 \mathrm{~h}$, the weakening trend of ACE2 in AngII + LPS group was also inferior to LPS group. These results showed that the change of ACE2 mRNA expression fell behind with the change of activity. ACE2 showed the minimum activity in both LPS and AngII + LPS groups at $6 \mathrm{~h}$. In addition, the results of immunofluorescence showed that the number of nuclei and the ACE2 expression were decreased in LPS groups at $6 \mathrm{~h}$, which could be further reduced by adding AngII (Fig. 2D).

The consumption of ACE2 in LPS-induced BEAS2B cells indicated that AngII may function as an enhancer of lung inflammation. To test this hypothesis, we investigated whether inhibition of AngII affects inflammation and cell apoptosis in bronchial epithelial BEAS-2B cells treated with LPS. After 

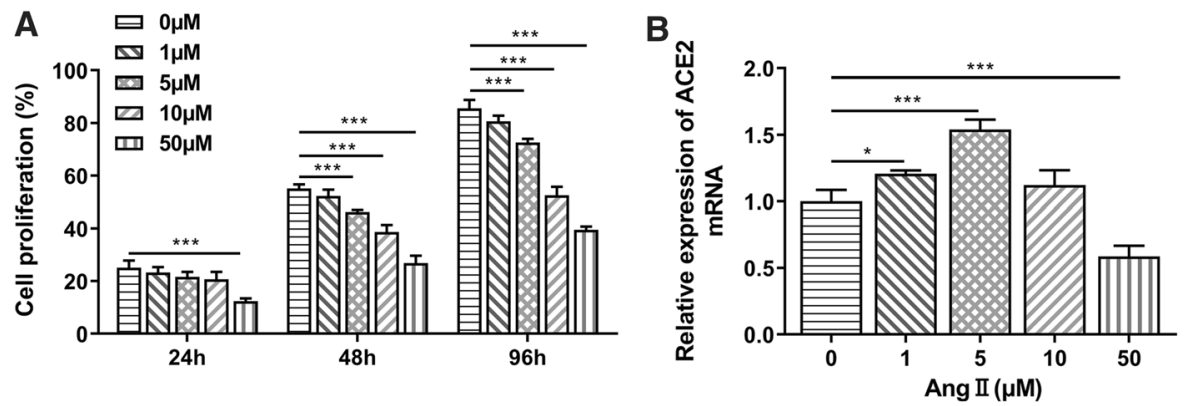

Fig. 1 After human bronchial epithelial cells underwent the treatment of different concentration of AngII $(0,1,5,10,50$ $\mu \mathrm{mol} / \mathrm{L})$, the detection of cell proliferation through CCK-8 assay

(A) and the detection of ACE2 mRNA through RT-qPCR

(B) were performed, respectively. ${ }^{*} \mathrm{p}<0.05$ and $* * * \mathrm{p}<0.001$

A
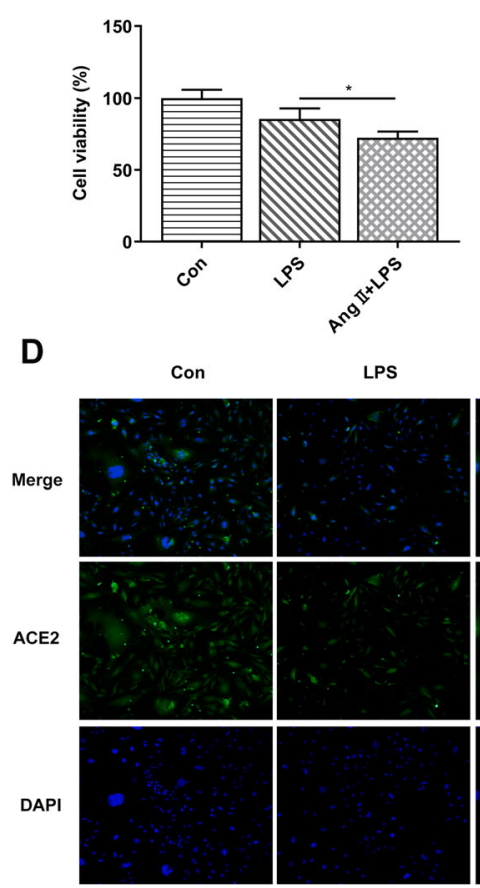

B

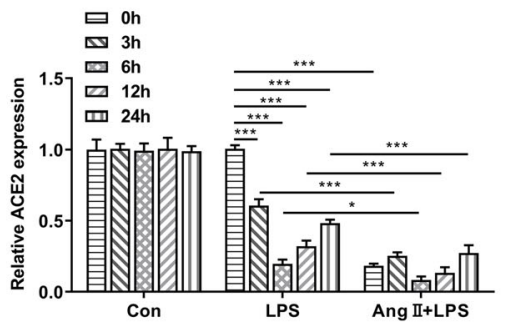

E

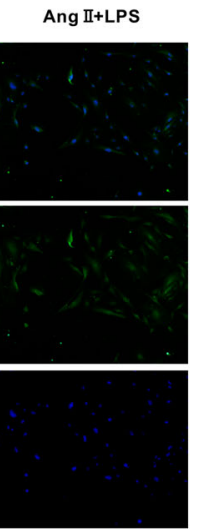

C
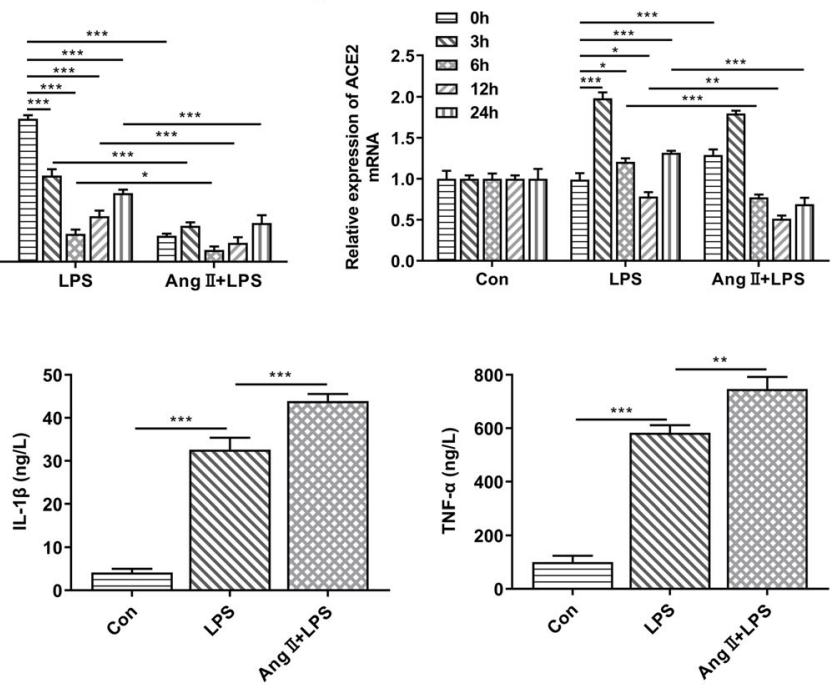

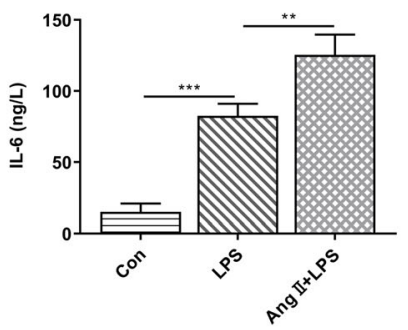

Fig. 2 After LPS or AngII treatment, human bronchial epithelial cells were used to perform the detection of cell viability through CCK-8 assay (A), the analysis of ACE2 activities (B) and the measurement of ACE2 mRNA through

$24 \mathrm{~h}$, in Fig. 2E, the proinflammatory cytokines, including IL-1 $\beta$, IL-6 and TNF- $\alpha$, induced by LPS were all significantly enhanced by AngII. Further, in western blot assay (Fig. 3A, B), compared with control group, the expression of Bax, cleaved-caspase-3 and ACE2 were upregulated, and the

qPCR $(\mathbf{C})$, immunofluorescence assay for the analysis of ACE2 expression (D), and qPCR assay for the analysis of expression of IL-1 $\beta$, IL-6 and TNF- $\alpha$ (E). $* \mathrm{p}<0.05$, * $* 0.01$ and $* * * \mathrm{p}<0.001$

expression of Bcl-2 was downregulated, and these tendencies could be further strengthened by adding AngII. These results indicate that AngII enhances proinflammatory cytokine production and cell apoptosis induced by LPS. 
A
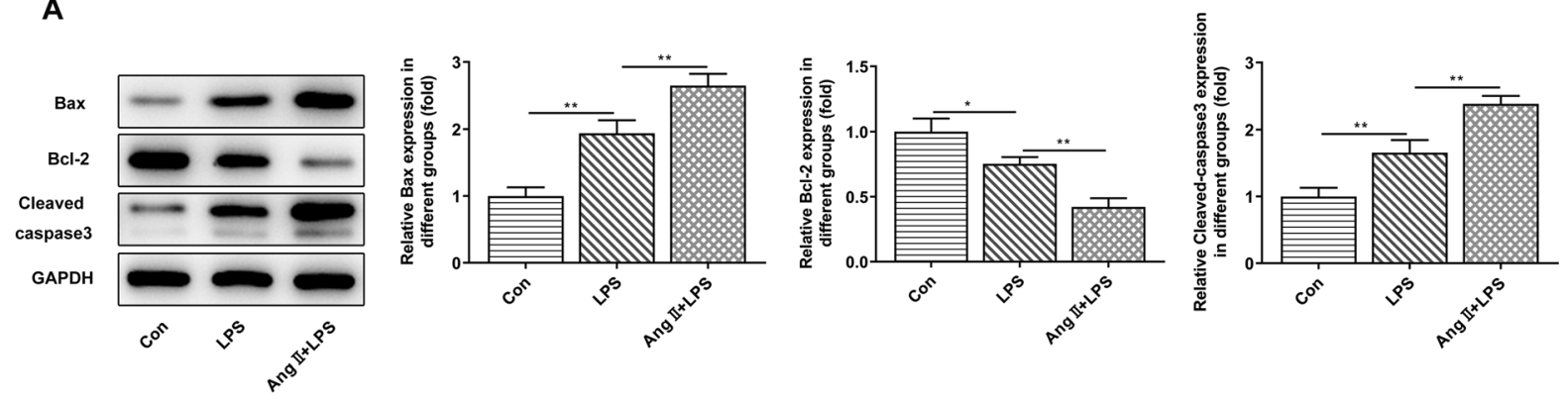

B
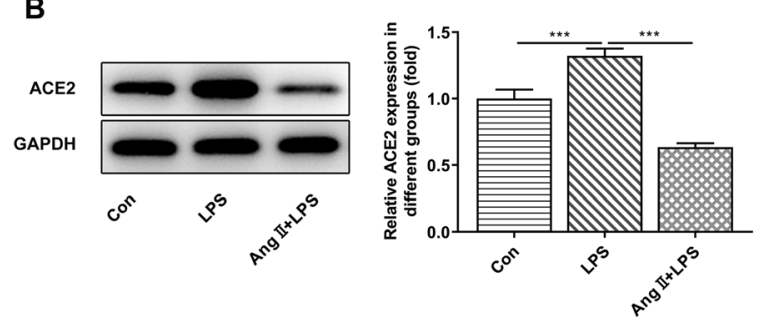

Fig. 3 After LPS or AngII stimulation, human bronchial epithelial cells were used to perform western blot assay for the expression analysis of Bax, cleaved-caspase-3 and Bcl-2

AngII promotes LPS-induced inflammation by regulating miR-143 in BEAS-2B cells

miRNAs are important regulators in lung inflammation. To further verify the underlying mechanism of the effect of AngII in LPS-induced BEAS-2B cells, we utilized multiple target prediction programs to identify the potential miRNAs that ACE2 targets. miR-143-3p was highlighted for further investigation.

RT-PCR was performed to investigate the expression of miR-143-3p in BEAS-2B cells, shown in Fig. 3C. We observed an increase in miR-143-3p expression in LPS-induced BEAS-2B cells compared with control group, which could be enhanced by AngII. In addition, a transfection plasmid, miR-143$3 p$ inhibitor or negative control RNAs (miR-NC) was transfected to BEAS-2B cells. Compared with the control and the miR-NC groups, the expression of miR-143-3p was significantly decreased in miR-143$3 p$ inhibitor group (Fig. 4A).

Next, we investigated whether miR-143-3p is critical for the function of AngII in BEAS-2B cells. CCK-8 assay showed that AngII pretreatment significantly inhibited the viability of LPS-induced BEAS$2 \mathrm{~B}$ cells, and that this inhibitory effect was reversed by miR-143-3p inhibitor transfection (Fig. 4B). ACE2

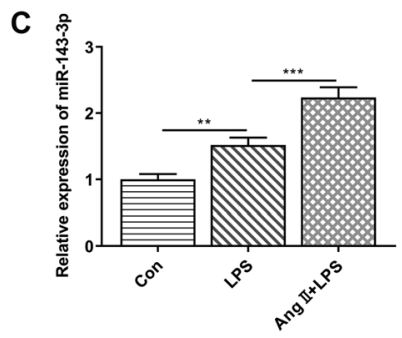

(A) and ACE (B), and qPCR for the analysis of miR-143-3p levels $(\mathbf{C})$. $* \mathrm{p}<0.05, * * \mathrm{p}<0.01$ and $* * * \mathrm{p}<0.001$

activity was significantly decreased in AngII + LPS and AngII + LPS + NC groups compared with control group in $6 \mathrm{~h}$, and this tendency could be reversed by transfecting miR-143-3p inhibitor (Fig. 4C). The expressions of proinflammatory cytokines, including IL-1 $\beta$, IL- 6 and TNF- $\alpha$, induced in AngII + LPS and AngII + LPS + NC groups were reversed by miR-143-3p inhibitor. Western blot assay (Fig. 4D) showed that compared with the control group, the expressions of Bax, cleaved-caspase- 3 and ACE2 were upregulated while that of Bcl-2 was downregulated in AngII + LPS and AngII + LPS + $\mathrm{NC}$ groups, and that these tendencies could be reversed by adding miR-143-3p inhibitor (Fig. 5A, B).

The dual-luciferase reporter assay confirmed the relationship between ACE2 and miR-143-3p predicted by bioinformatics methods. Firstly, RT-PCR showed increased expression of miR-143-3p in AngII and LPS-induced BEAS-2B cells compared with control group, which could be reversed by miR-143-3p inhibitor (Fig. 5C). Next, dual-luciferase reporter results showed that cells co-transfected with pDNAACE2-wild type and the miR-143-3p mimic (the wtACE2 + miR-143-3p mimic group) had significantly lower luciferase activity than cells co-transfected with pDNA-ACE2-wild type and the miR-NC (the wt- 

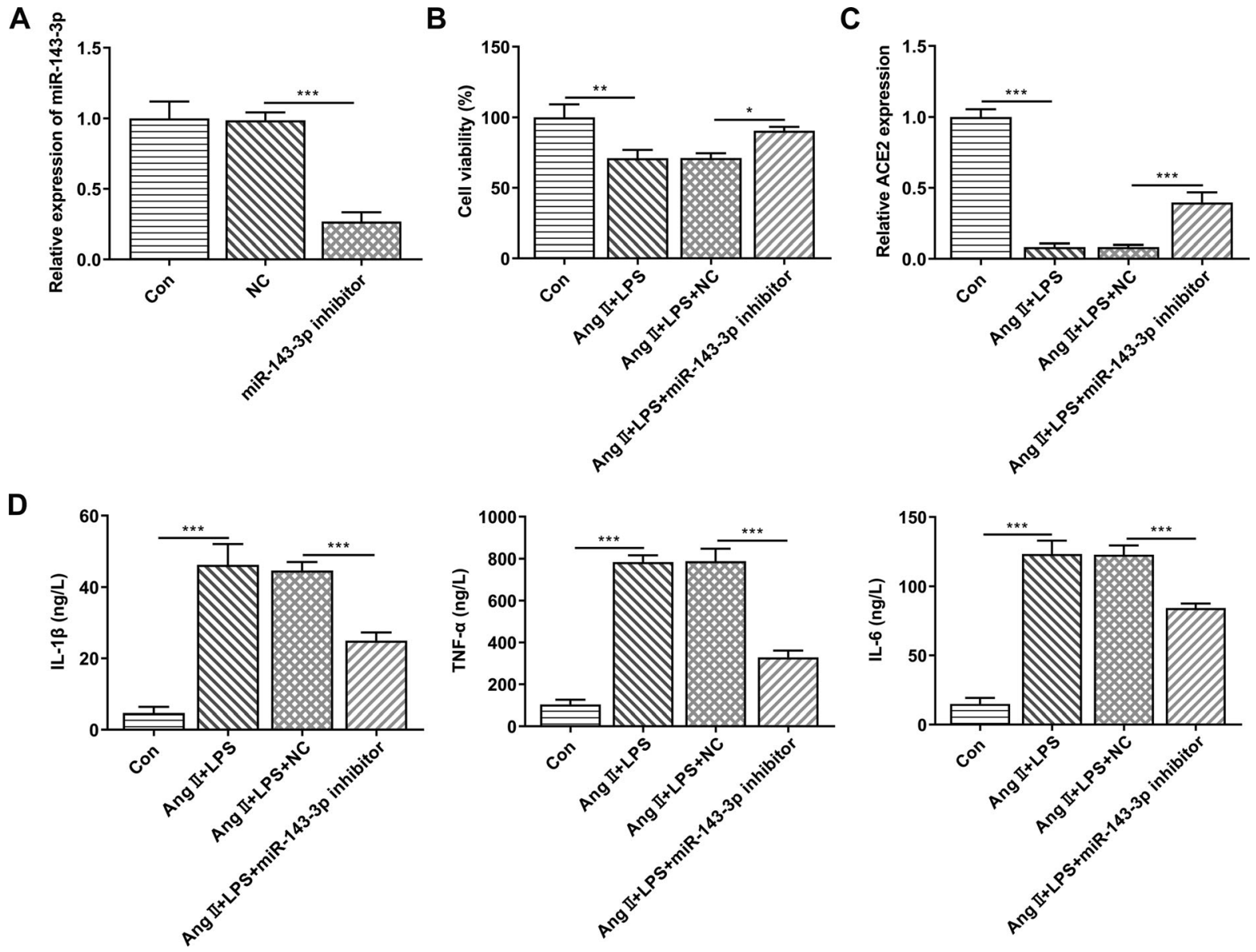

Fig. 4 miR-143-3p inhibitor interfered the effects of AngII on inflammatory markers in BEAS-2B cells under LPS stimulation. The analysis of miR-143-3p expression through qPCR (A), cell

ACE2 + NC mimic group). In contrast, we found no significant difference in the relative luciferase activity between the mut-ACE2 + miR-143-3p and the mutACE2 + NC mimic groups (Fig. 5E). These data strongly suggest that ACE2 targets and has a negative correlation with miR-143-3p expression in lung inflammation.

\section{Discussion}

Pneumonia has been regarded as a leading cause of infectious disease-related morbidity and mortality worldwide (Ravi Kumar et al. 2018). In our study, we used LPS and AngII to establish the bronchial cell inflammatory injury model. We found that $1 \mathrm{mg} / \mathrm{L}$ LPS and $5 \mu \mathrm{mol} / \mathrm{L}$ AngII treatment successfully viability through CCK-8 assay (B), ACE2 activities (C), the detection of IL-1 $\beta$, IL- 6 and TNF- $\alpha$ levels through ELISA (D). $* \mathrm{p}<0.05, * * \mathrm{p}<0.01$ and $* * * \mathrm{p}<0.001$

induced BEAS-2B cell apoptosis, inhibited cell viability, and enhanced the expression of proinflammatory cytokines. In this study, the important role of miR-143-3p in lung inflammation is identified. miR143-3p expression upregulated in AngII and LPSinduced injury induced cell apoptosis and inflammation and decreased the expression and the activity of ACE2 by targeting it in AngII and LPS-induced BEAS-2B cells. Therefore, miR-143-3p may be a promising therapeutic target for pneumonia treatment.

ACE2, whose function can be generally explained by its name, converts angiotensin and thereby regulates the renin-angiotensin system, RAS (Mowry et al. 2019). RAS regulates blood pressure, fluid dynamics, and electrolyte balance. Additionally, it plays a role in modulating the innate immune system and is a potent regulator of inflammation (Sodhi et al. 2019). In our 


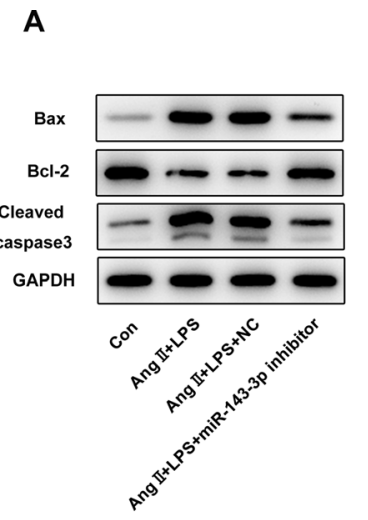

B

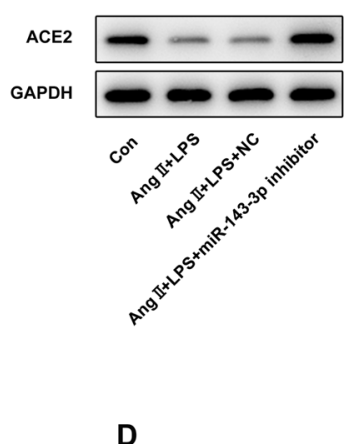

ACE2: 5' aggugcuugaucaaCAUCUCc 3' IIIIII

hsa-miR-143-3p: 3' cucgaugucacgaaGUAGAGu 5 ,
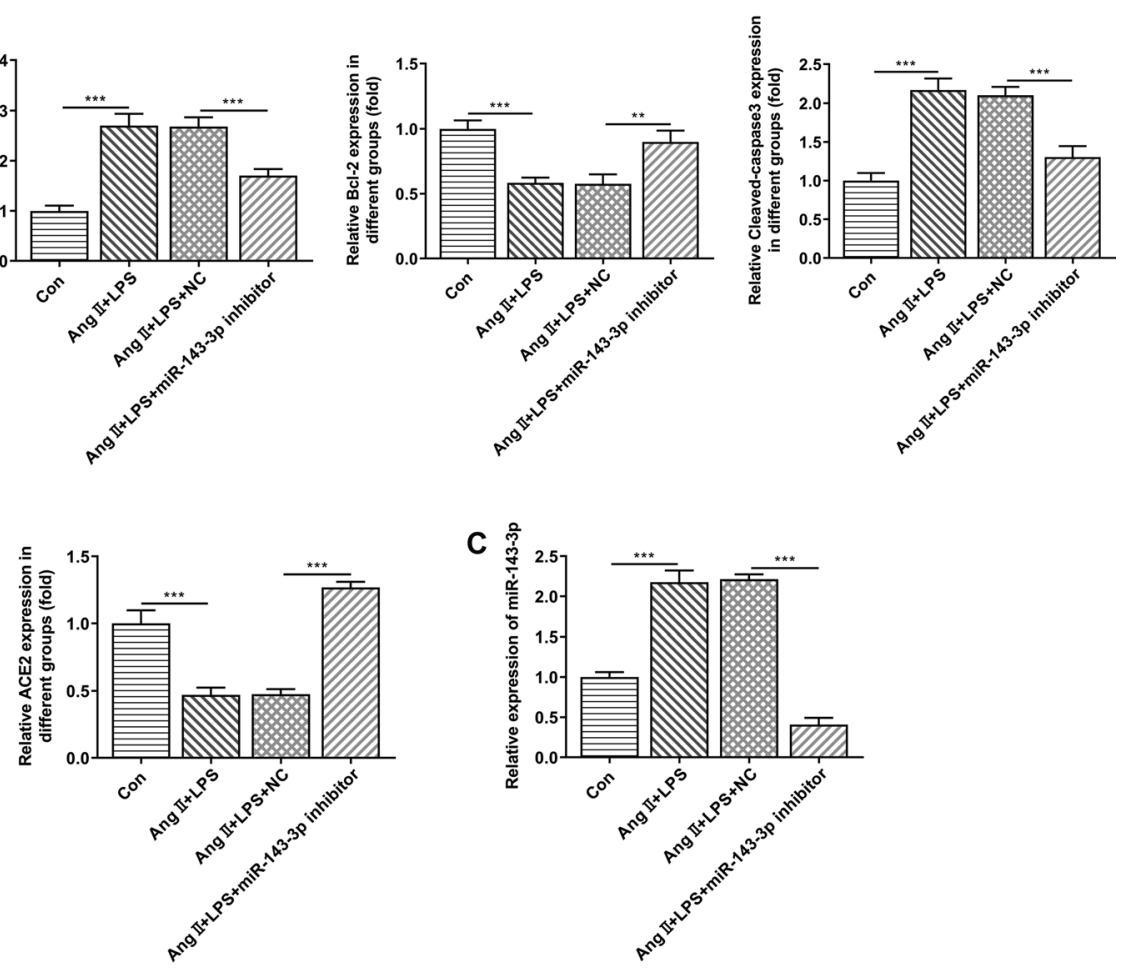

C

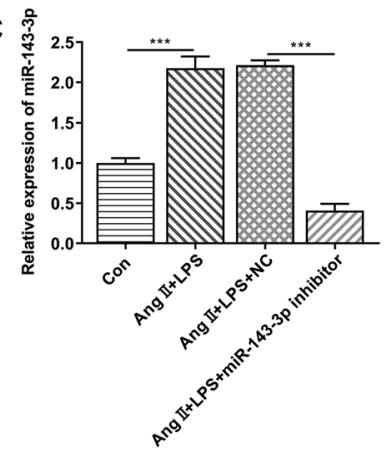

E

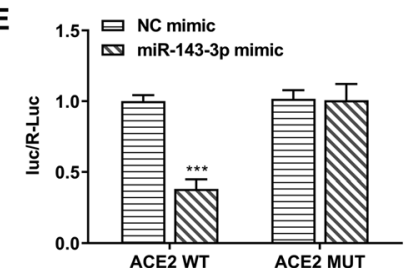

prediction of binding sites between ACE2 and miR-143-3p (D) and dual-luciferase reporter assay (E). ${ }^{* *} \mathrm{p}<0.01$ and *** $\mathrm{p}<0.001$ The detection of Bax, cleavedcaspase-3, Bcl-2 and ACE2 proteins through western blot (A, B), the analysis of miR-143-3p mRNA through qPCR (C), the

study, ACE2 expression and activity were timedependently decreased in LPS-induced group compared with the control group within the first $12 \mathrm{~h}$, which started to increase after $12 \mathrm{~h}$. Pretreatment of LPS significantly increased the expression of proinflammatory cytokines, enhanced cell apoptosis and regulated the expression of Bax, Bcl-2 and cleaved caspase-3 in BEAS-2B cells. Again, in view of ACE2's ability to convert AngII to Ang 1-7, blocking AngII/AGT1R and activating Ang 1-7/Mas1, it thereby alleviates the inflammatory response induced by RAS. (Shi et al. 2013). Moreover, Ang 1-7 can act against AngII and dampen its effects (Liu et al. 2016). In our study, ACE2 expression was significantly increased after pretreatment of AngII in $3 \mathrm{~h}$ compared with LPS group, which might owe to the positive feedback of AngII. In fact, in other diseases such as sepsis and acid aspiration-induced lung injury, ACE2 has been shown to be beneficial for modulating the inflammatory response. And studies have shown that pulmonary ACE2 is impaired in several lung diseases, including viral and aspiration pneumonia, pulmonary hypertension, and LPS-induced lung injury (Imai et al. 2005; Shao et al. 2019). Additionally, ACE2, which was abundantly expressed in the lung cells including vascular endothelium and respiratory epithelium, could mediate the access of SARS-CoV-2 to host cells (Amraei et al. 2020; Sardu et al. 2020; Pearce 
et al. 2020). Systemic inflammatory response caused by SARS-CoV-2 is the most important pathological basis in novel coronavirus pneumonia patients, especially in critical patients who died due to complicated sepsis. Recently, a study proposed that the decreased ACE2 receptor expression was engaged in keeping off SARS-CoV-2 invasion of pulmonary epithelial cell (Hasanagic et al. 2020). Our work suggested that ACE was reduced by AngII via miR-143-3p, which provides a new sight for into pathogenesis of COVID-19 induced lung inflammation. Latest studies revealed that pneumonia and lung cancer are significant hazard factors connected to the mortality of patients of certain age with hypertension (Shen et al. 2016). Hypertension is commonly seen in non-cardiovascular diseases, including severe Coronavirus Disease 2019 (COVID19) pneumonia and acute respiratory distress syndrome, ARDS (Pranata et al. 2020). Interestingly, in our study, the ACE2 expression was largely consumed in AngII + LPS group between 3 and $12 \mathrm{~h}$, which was consistent with prior studies and might explain the relationship between hypertension and lung inflammation.

miRs are small non-coding RNAs, which are approximately 19 to 25 nucleotides long and act as posttranscriptional regulators of gene expression (Lagos-Quintana and Lagos-Quintana 2001). A large number of studies have proven that miRNAs can regulate various cellular processes such as differentiation, proliferation, and cell-cell interaction (Chen and Meister 2005). Likewise, some miRNAs are dysregulated in lung inflammation and functionally implicated in the pathogenesis of lung inflammation (Xue et al. 2018; Yun et al. 2020). In previous study, serum miR-143 was found to be increased in patients suffering from sepsis and thought to be a potentially valuable biomarker for distinguishing sepsis and systemic inflammatory response syndrome, SIRS (Han et al. 2016). Decreased expression of miR-143$3 p$ has been observed in fibromyalgia, further suggesting its association with inflammatory pain (Wang et al. 2020). Our results showed that pretreatment of AngII and LPS regulated the activity of ACE2, increased the expression of proinflammatory cytokines, and promoted cell apoptosis in BEAS-2B cells, which could be reversed by transfection of miR-143$3 p$ inhibitor. Above all, the present study revealed that AngII promotes LPS-induced inflammation by regulating miR-143-3p in BEAS-2B cells.
In conclusion, our results proved that miR-143-3p inhibits ACE2 by targeting the $3^{\prime}$-UTR of ACE2. Functional experiments in our study showed that AngII promotes apoptosis and inflammation by regulating miR-143-3p in BEAS-2B cells. These results suggest that the regulation of ACE2 by miR-143-3p may become a novel strategy for the treatment of lung inflammation.

Funding The study was supported by "Study on the role of Th17 and its cytokines in AECOPD induced by rhinovirus" [No. 2018FE001(-292)] and "Study on the H19/Mir-29b-3P/ HGMB1/TLR4 signaling pathway of ginsenoside in the treatment of lung injury caused by smoking" [No. 909016590033].

\section{Declarations}

Conflict of interest The authors declare no conflict of interest.

\section{References}

Ahmad S et al (2019) Transcriptome meta-analysis deciphers a dysregulation in immune response-associated gene signatures during sepsis. Genes (Basel) 10:1005

Ahmad S et al (2020) Identification and validation of potential miRNAs, as biomarkers for sepsis and associated lung injury: a network-based approach. Genes (Basel) 11:1327

Amraei R, Rahimi N (2020) COVID-19, renin-angiotensin system and endothelial dysfunction. Cells 9:1652

Arora M et al (2011) LPS-induced CD11b+Gr1(int)F4/80+ regulatory myeloid cells suppress allergen-induced airway inflammation. Int Immunopharmacol 11:827-832

Bartel DP (2009) MicroRNAs: target recognition and regulatory functions. Cell 136:215-233

Boettger T et al (2009) Acquisition of the contractile phenotype by murine arterial smooth muscle cells depends on the mir143/145 gene cluster. J Clin Investig 119:2634-2647

Boskabadi J et al (2019) Immunomodulatory properties of captopril, an ACE inhibitor, on LPS-induced lung inflammation and fibrosis as well as oxidative stress. Inflammopharmacology 27:639-647

Chen PY, Meister G (2005) microRNA-guided posttranscriptional gene regulation. Biol Chem 386:1205-1218

Gallicano GI et al (2020) Molecular targeting of vulnerable RNA sequences in SARS CoV-2: identifying clinical feasibility. Gene Ther. https://doi.org/10.1038/s41434-02000210-0

Han Y et al (2016) Diagnostic value of elevated serum miRNA143 levels in sepsis. J Int Med Res 44:875-881

Hasanagic S, Serdarevic F (2020) Potential role of memantine in the prevention and treatment of COVID-19: its antagonism of nicotinic acetylcholine receptors and beyond. Eur Respir J 56:2001610

Hu X et al (2019) Neutralization of interleukin-17A attenuates lipopolysaccharide-induced mastitis by inhibiting 
neutrophil infiltration and the inflammatory response. J Interferon Cytokine Res 39:577-584

Imai $Y$ et al (2005) Angiotensin-converting enzyme 2 protects from severe acute lung failure. Nature 436:112-116

Khair OA, Davies RJ, Devalia JL (1996) Bacterial-induced release of inflammatory mediators by bronchial epithelial cells. Eur Respir J 9:1913-1922

Khan MJ et al (2020) Inhibition of miRNA-34a promotes M2 macrophage polarization and improves LPS-induced lung injury by targeting Klf4. Genes (Basel) 11:966

Kim VN, Han J, Siomi MC (2009) Biogenesis of small RNAs in animals. Nat Rev Mol Cell Biol 10:126-139

Lagos-Quintana M et al (2001) Identification of novel genes coding for small expressed RNAs. Science 294:853-858

Li W et al (2003) Angiotensin-converting enzyme 2 is a functional receptor for the SARS coronavirus. Nature 426:450-454

Li B et al (2011) Pulmonary epithelial CCR3 promotes LPSinduced lung inflammation by mediating release of IL-8. J Cell Physiol 226:2398-2405

Liu D et al (2016) Association between circulating levels of ACE2-Ang-(1-7)-MAS axis and ACE2 gene polymorphisms in hypertensive patients. Medicine 95:e3876

Livak KJ, Schmittgen TD (2001) Analysis of relative gene expression data using real-time quantitative PCR and the $2^{(- \text {Delta Delta C(T)) }}$ Method. Methods 25:402-408

Matarese A et al (2020) miR-98 regulates TMPRSS2 expression in human endothelial cells: key implications for COVID19. Biomedicines 8:462

Mowry FE, Biancardi VC (2019) Neuroinflammation in hypertension: the renin-angiotensin system versus proresolution pathways. Pharmacol Res 144:279-291

Pearce L, Davidson SM, Yellon DM (2020) The cytokine storm of COVID-19: a spotlight on prevention and protection. Expert Opin Ther Targets 24:723-730

Ponsuksili S et al (2017) Genetic architecture and regulatory impact on hepatic microRNA expression linked to immune and metabolic traits. Open Biol 7:170101

Pranata R et al (2020) Hypertension is associated with increased mortality and severity of disease in COVID-19 pneumonia: a systematic review, meta-analysis and meta-regression. J Renin Angiotensin Aldosterone Syst 21:14703203209 26899

Ravi Kumar S et al (2018) Emerging roles of inflammasomes in acute pneumonia. Am J Respir Crit Care Med 197:160-171

Sardu C et al (2020) Hypertension, thrombosis, kidney failure, and diabetes: is COVID-19 an endothelial disease? A comprehensive evaluation of clinical and basic evidence. J Clin Med 9:1417
Shao M et al (2019) Exogenous angiotensin (1-7) directly inhibits epithelial-mesenchymal transformation induced by transforming growth factor-beta1 in alveolar epithelial cells. Biomed Pharmacother 117:109193

Shen Y et al (2016) Impact of pneumonia and lung cancer on mortality of women with hypertension. Sci Rep 6:20

Shi Y et al (2013) Osthole protects lipopolysaccharide-induced acute lung injury in mice by preventing down-regulation of angiotensin-converting enzyme 2. Eur J Pharm Sci 48:819-824

Silva GM et al (2020) Role of renin-angiotensin system components in atherosclerosis: focus on Ang-II, ACE2, and Ang-1-7. Front Physiol 11:1067

Sodhi CP et al (2018) Attenuation of pulmonary ACE2 activity impairs inactivation of des-Arg(9) bradykinin/BKB1R axis and facilitates LPS-induced neutrophil infiltration. Am J Physiol Lung Cell Mol Physiol 314:L17-L31

Sodhi CP et al (2019) A dynamic variation of pulmonary ACE2 Is required to modulate neutrophilic inflammation in response to Pseudomonas aeruginosa lung infection in mice. J Immunol 203:3000-3012

Tang H et al (2020) The noncoding and coding transcriptional landscape of the peripheral immune response in patients with COVID-19. Clin Transl Med 10:e200

Wang YS et al (2014) Role of miR-145 in cardiac myofibroblast differentiation. J Mol Cell Cardiol 66:94-105

Wang Y et al (2020) miR-143-3p impacts on pulmonary inflammatory factors and cell apoptosis in mice with mycoplasmal pneumonia by regulating TLR4/MyD88/NFkappaB pathway. Biosci Rep. https://doi.org/10.1042/ bsr20193419

Xue H, Li MX (2018) MicroRNA-150 protects against cigarette smoke-induced lung inflammation and airway epithelial cell apoptosis through repressing p53: microRNA-150 in CS-induced lung inflammation. Hum Exp Toxicol 37:920-928

Yun J et al (2020) Up-regulation of miR-297 mediates aluminum oxide nanoparticle-induced lung inflammation through activation of Notch pathway. Environ Pollut 259:113839

Zhang YH et al (2015) ACE2 and Ang-(1-7) protect endothelial cell function and prevent early atherosclerosis by inhibiting inflammatory response. Inflamm Res 64:253-260

Publisher's Note Springer Nature remains neutral with regard to jurisdictional claims in published maps and institutional affiliations. 